\title{
A NOTE ON GENERATORS OF EVEN-NUMBERED $K$-GROUPS OF RINGS OF INTEGERS
}

\author{
Wojciech Gajda And Cornelius Greither
}

\begin{abstract}
In this note we give explicit generators for étale even-numbered $K$-groups of the ring of integers in some cyclotomic fields, using generalised symbols and standard results in cyclotomic Iwasawa theory.
\end{abstract}

\section{Introduction}

In this short note we intend to give explicit generators for étale even-numbered $K$-groups of the ring of integers in some cyclotomic fields. These $K$-groups are finite, often nontrivial, and interesting; for example, $K_{2 n}^{e t}\left(\mathbb{Z}\left[\frac{1}{l}\right]\right)$, for an odd natural number $n$ and an odd prime $l$, is expected to be cyclic, and this cyclicity is equivalent to the Iwasawa conjecture on cyclicity of the minus part of the $l$-Sylow subgroup of the class group of the cyclotomic field $\mathbb{Q}\left(\mu_{l}\right)$, see for instance [Ga] or $[\mathrm{Ku}]$.

Unpublished work of Voevodsky and Rost implies that the étale $K$-groups agree with the corresponding algebraic $K$-groups of Quillen, in the $l$-part for every odd prime $l$, see for instance Theorem 70 in [We]. However we prefer to stay within the étale cohomology framework throughout this paper.

The generators of the $K$-groups which we construct arise as images under transfer of certain multiplicative combinations of generalised symbols. Indeed, for $K_{2}$ we do have symbols in the usual sense. The generalised symbols that we use always involve non-units and therefore only lie in the $K$-group of a certain field, and the whole point is to choose the multiplicative combinations of them in such a way that we get all elements of the $K$-group of the appropriate ring of integers, and nothing else.

Our final result (Theorem 3.2) does not make much sense without the constructions which go into it, so we choose not to describe it in full detail here. For the ease of the reader, the construction is first explained in a special setting in Section 2 (the main result of that section is Proposition 2.3). A reader just interested in the final statement is invited to skip Section 2 completely (at least at the first reading).

The techniques should certainly work in greater generality, but we decided to keep the setting simple and very explicit. We use some well-known results on Stickelberger elements and a fairly simple-minded algebraic Lemma 3.1. Even though we use class groups in our proofs, it does not seem that our methods will yield results on class groups; they only work for higher $K$-groups.

Received by the editors October 31, 2008.

Key words and phrases. Class group, cyclotomic field, étale cohomology, $K$-groups. 


\section{The setting, and a special case of our construction}

Let $l$ be an odd prime, let $F=\mathbb{Q}\left(\mu_{l}\right)$ and $F^{+}=\mathbb{Q}\left(\mu_{l}\right)^{+}$be the cyclotomic field and its maximal real subfield, respectively. We also consider the cyclotomic field $E=\mathbb{Q}\left(\mu_{l^{k}}\right)$, where the exponent $k$ will be specified below. Let $n$ be an odd natural number. In what follows, let $S$ denote a finite set of finite primes of $F$; however, in context, this will also indicate the set of primes of $E$ above that finite set. In this note we will identify the étale K-theory groups $K_{2 n}^{e t}\left(\mathcal{O}_{L, S}, \mathbb{Z} / l^{k}\right)$ and $K_{2 n+1}^{e t}\left(\mathcal{O}_{L, S}, \mathbb{Z} / l^{k}\right)$, of the ring of $S$-integers of a number field $L$ (where $S$ contains the primes of $L$ over $l)$, with the étale cohomology groups $\mathrm{H}^{2}\left(\mathcal{O}_{L, S}, \mathbb{Z} / l^{k}(n+1)\right)$ and $\mathrm{H}^{1}\left(\mathcal{O}_{L, S}, \mathbb{Z} / l^{k}(n+1)\right)$, respectively via the Dwyer-Friedlander isomorphisms, which respect transfers for finite field extensions and preserve the multiplicative structure (cf. [DF]). We will also use identifications: $K_{2 n}^{e t}\left(\mathcal{O}_{L, S}\right)=\mathrm{H}^{2}\left(\mathcal{O}_{L, S}, \mathbb{Z}_{l}(n+1)\right)$ and $K_{2 n+1}^{e t}\left(\mathcal{O}_{L, S}\right)=\mathrm{H}^{1}\left(\mathcal{O}_{L, S}, \mathbb{Z}_{l}(n+1)\right)$, which follow from the Dwyer-Friedlander isomorphisms by passing to the inverse limits over $k$. By definition $K_{2 n}^{e t}\left(\mathcal{O}_{F, S}\right)\left[l^{k}\right]$ is the $l^{k}$-torsion part of $\mathrm{H}^{2}\left(\mathcal{O}_{F, S}, \mathbb{Z}_{l}(n+1)\right)$.

Consider the homomorphism

$$
\alpha: \mathcal{O}_{E, S}^{\times} \otimes \mathbb{Z} / l^{k}(n) \longrightarrow K_{2 n}^{e t}\left(\mathcal{O}_{F, S}\right)\left[l^{k}\right]
$$

which we define using the cohomological construction of the étale $K$-group. There is a map $\alpha^{\prime}$ :

$$
\mathcal{O}_{E, S}^{\times} \otimes \mathbb{Z} / l^{k}(n) \rightarrow \mathrm{H}^{1}\left(\mathcal{O}_{E, S}, \mathbb{Z}_{l}(1)\right) \otimes \mathrm{H}^{0}\left(\mathcal{O}_{E, S}, \mathbb{Z} / l^{k}(n)\right) \rightarrow \mathrm{H}^{1}\left(\mathcal{O}_{E, S}, \mathbb{Z} / l^{k}(n+1)\right),
$$

where the first map is standard in the first tensor factor (and trivial in the other), and the second map is the cup product. To obtain $\alpha$, we then follow up this map with the canonical epimorphism $\mathfrak{b}$ (the Bockstein map) from the group $\mathrm{H}^{1}\left(\mathcal{O}_{E, S}, \mathbb{Z} / l^{k}(n+1)\right)$ to $\mathrm{H}^{2}\left(\mathcal{O}_{E, S}, \mathbb{Z}_{l}(n+1)\right)\left[l^{k}\right]$, and then with the transfer map down to the analogous group with $E$ replaced by $F$. (Of course one could interchange the Bockstein map and transfer, in the obvious sense.)

The main result of this section is Prop. 2.3 which shows that the étale evennumbered $K$-groups of $\mathbb{Z}\left[\frac{1}{l}\right]$ can be described by means of the map $\alpha_{\mathbb{Q}}$ defined as $\alpha$ followed by transfer from $F$ to $\mathbb{Q}$. To this end we have to place $\alpha_{\mathbb{Q}}$ into a certain commutative diagram. Before we do this, let us give a comparison result, to put the map $\alpha$ into context.

Lemma 2.1. The above definition of the map $\alpha$ gives the same map as the construction in Lemma 2.3 of [BG1] (where in loc. cit. we replace $\mathbb{Q}$ by F). 
Proof: Consider the following diagram

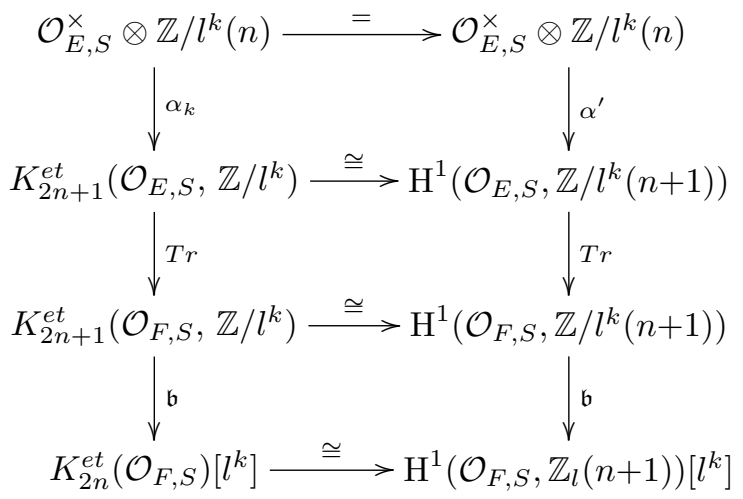

where the composition of vertical maps on the left hand side is the map of Lemma 2.3 in [BG1], and the composition of vertical maps on the right hand side is the cohomological counterpart of the map $\alpha$. The horizontal maps in the diagram are the Dwyer-Friedlander isomorphisms. The map $\alpha_{k}$ was defined in [BG1] as the composition of $\alpha^{\prime}$ with the inverse of the Dwyer-Friedlander isomorphism. This makes the upper square commute. Commutativity of the lower squares follows, since the Dwyer-Friedlander isomorphisms commute with transfers and with the connecting homomorphisms $\mathfrak{b}$ in K-theory and étale cohomology.

Recall from the paper [BG1] that the map $\alpha$ is surjective if the norm map

$$
\mathrm{N}_{E / F}: \mathcal{O}_{E, S}^{\times} \longrightarrow \mathcal{O}_{F, S}^{\times}
$$

is onto, for which it suffices that the set $S$ contains the prime over $l$ and a set of primes $\mathfrak{p}$ of degree one of $\mathcal{O}_{F}$ such that the primes $\mathfrak{q}$ above these $\mathfrak{p}$ generate the class group of $E$.

We will need the Galois groups $G=\operatorname{Gal}(E / F), \tilde{G}=\operatorname{Gal}(E / \mathbb{Q})$, and $\Delta=$ $\operatorname{Gal}(F / \mathbb{Q})$. Note that $\tilde{G}=G \times \Delta$.

Let us treat a particularly simple situation in this section: we assume until the end of the section that the minus part of the $l$-part of the class group of $\mathcal{O}_{F}$ is cyclic as a $\mathbb{Z}_{l}[\Delta]$-module, hence the minus part of the $l$-part of the class group $\mathcal{O}_{E}$ with $k \geq 1$ is cyclic over $\mathbb{Z}_{l}[\tilde{G}]$ (codescent plus Nakayama's Lemma). Take a Galois module generator $\mathfrak{q}$ of $C l\left(\mathcal{O}_{E}\right)\{l\}$ (the $l$-part of the class group of $\mathcal{O}_{E}$ ) and let $S$ consist of the prime $\mathfrak{p}$ below $\mathfrak{q}$ and the prime above $l$. The standard Iwasawa module $X_{F}^{-}$is then cyclic over $\Lambda=\mathbb{Z}_{l}[G][\Delta]$, and isomorphic to $\Lambda_{-} /\left(\Theta_{*}\right)$, where $\Theta_{*}$ is the Stickelberger element multiplied by a generator of the principal $\Lambda$-ideal $A n n\left(\mu_{l}\right)$, and the subscript minus at $\Lambda$ means modding out by $1+j$ (with $j$ meaning complex conjugation). (Note: We will later use $\Theta_{*}$, or rather its image in $\mathbb{Z}_{l}[\tilde{G}]$, as an exponent; in order for this to make sense, one simply takes a sufficiently good approximation in $\mathbb{Z}[\tilde{G}]$. All our arguments are carried out modulo a high $l$-power.)

We now let $\alpha_{\mathbb{Q}}$ be $\alpha$ followed by the transfer from $F$ to $\mathbb{Q}$. Then $\alpha_{\mathbb{Q}}$ is again onto by a standard argument, and this remains so if we take the plus part of its domain:

$$
\alpha_{\mathbb{Q}}: \mathcal{O}_{E, S}^{\times-} \otimes \mathbb{Z} / l^{k}(n) \longrightarrow K_{2 n}^{e t}\left(\mathcal{O}_{\mathbb{Q}, S}\right)\left[l^{k}\right] .
$$


We now choose $k$ such that $l^{k}$ is the number of elements of the group $K_{2 n}^{e t}\left(\mathbb{Z}\left[\frac{1}{l}\right]\right)$. Let $q$ be the rational prime below $\mathfrak{p}$ and $\mathfrak{q}$. Then $l^{k}$ divides $q-1$, and the group $K_{2 n-1}^{e t}\left(\mathbb{F}_{q}\right)$ is of order $q^{n}-1$, and this is exactly divisible by some power $l^{m} \geq l^{k}$ depending only on $q$ and $n$. The map val in the commutative diagram (d1) below comes from the valuation map at every conjugate of $\mathfrak{q}$ (note that $\tilde{G}$ acts freely on the set of these conjugates), and $\partial$ comes from the localisation sequence:

$$
0 \rightarrow K_{2 n}^{e t}\left(\mathbb{Z}\left[\frac{1}{l}\right]\right)\left[l^{k}\right] \rightarrow K_{2 n}^{e t}\left(\mathbb{Z}\left[\frac{1}{l q}\right]\right)\left[l^{k}\right] \rightarrow K_{2 n-1}^{e t}\left(\mathbb{F}_{q}\right)\left[l^{k}\right]
$$

The map $c$ is just the epimorphism $\mathbb{Z} / l^{k}[\tilde{G}](n) \rightarrow \mathbb{Z} / l^{k}[\tilde{G}](n)_{\tilde{G}}$ followed by the canonical isomorphism identifying these coinvariants with $\mathbb{Z} / l^{k}$. Finally, the group $K_{2 n-1}^{e t}\left(\mathbb{F}_{q}\right)\{l\}$ is identified with the $\mathbb{F}_{q}$-rational points of $\mu_{l \infty}^{\otimes n}$ (note also that the superscript ${ }^{e t}$ can certainly be omitted here), and its $l^{k}$-torsion is visibly generated by $\zeta^{\otimes n}$, where $\zeta \in \mathbb{F}_{q}$ is the image of a chosen primitive $l^{k}$-th root of unity $\zeta_{l^{k}}$. The map $\beta$ sends $\zeta^{\otimes n}$ to $-1 \in \mathbb{Z} / l^{k}$ by definition; it is an isomorphism.

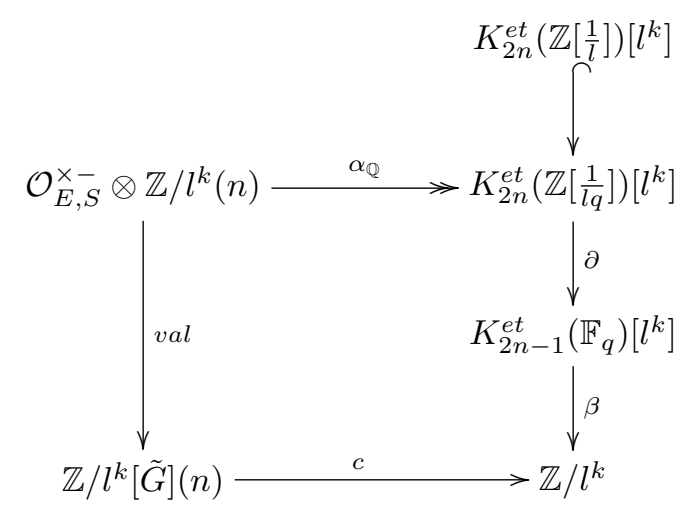

The kernel of $\partial$ is the image of the vertical inclusion map placed above it.

Lemma 2.2. The above diagram is commutative.

Proof: In the whole argument, we will identify $\mathbb{Z} / l^{k}(1)$ with $\mu_{l^{k}}$, where 1 maps to the chosen root $\zeta_{l^{k}}$ of unity. Then $\mathbb{Z} / l^{k}(n)$ is generated by 1 in additive notation (twist is hidden), or by $\zeta_{l^{k}}^{\otimes n}$ in multiplicative notation (twist is visible).

(1) For $n=1$ one can check that $\alpha_{\mathbb{Q}}\left(u \otimes \zeta_{l^{k}}\right)=\operatorname{Tr}_{E / \mathbb{Q}}\left\{u, \zeta_{l^{k}}\right\}$, where $\operatorname{Tr}$ stands for transfer, and inside the $\operatorname{Tr}$ term we have a Steinberg symbol in $K_{2}$. (It was shown by Tate that the Steinberg symbol corresponds to cup-product on the cohomological side, cf. Theorem 3.1 in [Ta]).

(2) Let $v$ be a place of $E$ above $q, E_{v}$ the $v$-adic completion, and $\mathbb{F}_{v}$ the residue field of $\mathcal{O}_{v}$, the integers of $E_{v}$. We look at a similar diagram in this local situation:

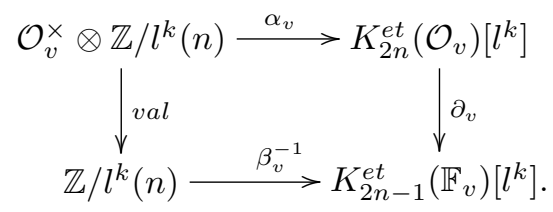

The isomorphism $\beta_{v}$ sends $\zeta^{\otimes n}$ to $-1 \in \mathbb{Z} / l^{k}(n)$. We claim this diagram commutes. Since the connecting homomorphism $\partial_{v}$ (which comes from the localisation sequence 
in étale cohomology) commutes with cup product, and from the definitions, we see that we only need to deal with the case $n=1$. (The case $n=0$ is excluded!) Here the upper horizontal map is given by symbols: $u \otimes \zeta_{l^{k}} \mapsto\left\{u, \zeta_{l^{k}}\right\}_{E_{v}}$, and $\partial_{v}$ is the tame symbol, mapping $\left\{u, \zeta_{l^{k}}\right\}_{E_{v}}$ to $\zeta_{l^{k}}^{-\operatorname{val}(u)}$, where val is the $v$-adic valuation map. From this, the commutativity of the diagram is clear.

(3) The lemma now follows from the compatibility of $\alpha$ and $\partial$ with localisation and transfer. To see this, the following diagram should be helpful ( $v$ always runs over the $E$-places above $q$; obvious localisation maps have no labels):

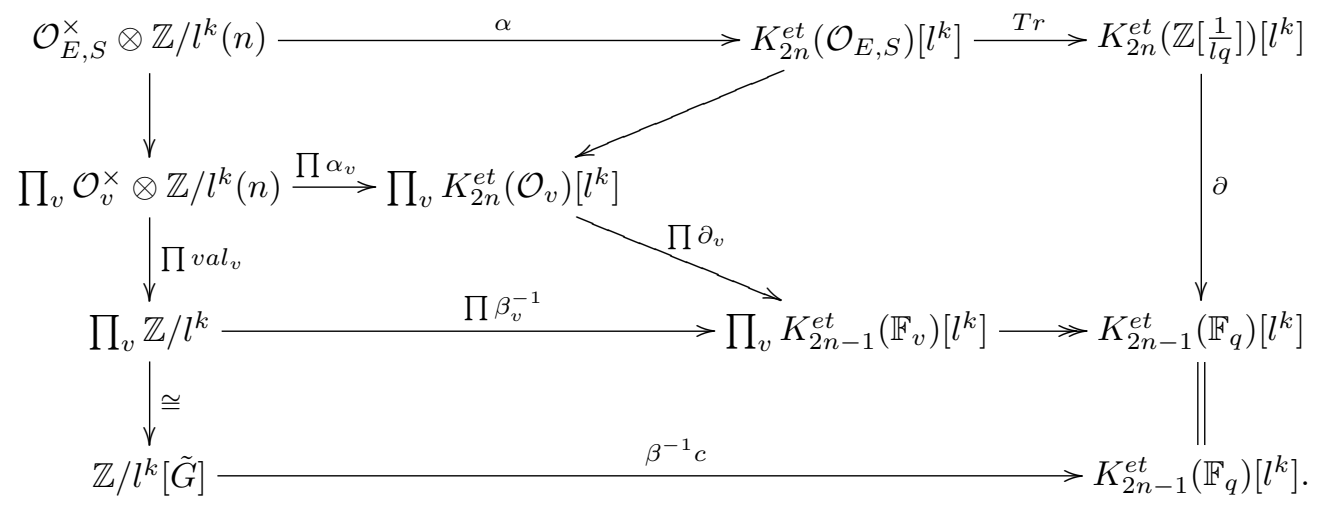

The right hand map in the third row is a norm map, more precisely on $K_{2 n-1}^{e t}\left(\mathbb{F}_{v}\right)\left[l^{k}\right]$ it is the canonical identification map wth $K_{2 n-1}^{e t}\left(\mathbb{F}_{q}\right)\left[l^{k}\right]$. The commutativity of the upper right hand pentagon can be taken from [Sou], p.276.

We now use Diagram (d1) to deduce an important property of the map $\alpha_{\mathbb{Q}}$.

Proposition 2.3. The image of the map $\alpha_{\mathbb{Q}}$ is already contained in $K_{2 n}^{e t}\left(\mathbb{Z}\left[\frac{1}{l}\right]\right)\left[l^{k}\right]$ and $\operatorname{im} \alpha_{\mathbb{Q}}=K_{2 n}^{\text {et }}\left(\mathbb{Z}\left[\frac{1}{l}\right]\right)$ is generated by $\alpha_{\mathbb{Q}}(x \otimes 1)$, where $x$ is a generator of $\mathfrak{q}^{\Theta_{*}}$.

ProOF: By Stickelberger's theorem we have indeed $\mathfrak{q}^{\Theta_{*}}=(x)$ for some $x \in \mathcal{O}_{E, S}^{\times}$. The left vertical map in the diagram $(\mathrm{d} 1)$ sends $x$ to $\bar{\Theta}_{*} \in \mathbb{Z} / l^{k}[\tilde{G}](n)^{+}$. Given the definition of the map $c$, this means that the composition of the left vertical and the lower horizontal map in Diagram $(\mathrm{d} 1)$ sends $x$ to the image of $\Theta_{*}$ under the composition:

$$
\Lambda \stackrel{\cong}{\longrightarrow} \Lambda(n) \stackrel{\varepsilon}{\longrightarrow} \mathbb{Z}_{l} \longrightarrow \mathbb{Z} / l^{k}
$$

Here $\varepsilon$ is just the canonical map identifying the $\operatorname{Gal}\left(\mathbb{Q}\left(\zeta_{l \infty}\right) / \mathbb{Q}\right)$ - invariants of $\Lambda(n)$ with $\mathbb{Z}_{l}$. Let us denote the first isomorphism by an exponent $(n)$. Then by the class number formula (cf. [BG2], Section 5), \# $K_{2 n}^{e t}\left(\mathbb{Z}\left[\frac{1}{l}\right]\right)=\left[\mathbb{Z}_{l}: \varepsilon \Theta_{*}^{(n)}\right]$, and the left hand side equals $l^{k}$ by assumption. Hence $\Theta_{*}$ is annihilated by the map in the last display, and therefore the composition of the left vertical and the lower horizontal maps of the diagram (d1) sends $x$ to zero. Since $\Theta_{*}$ is the precise annihilator of the classgroup of $E$, it follows that $\mathcal{O}_{E, S}^{\times-} \otimes \mathbb{Z} / l^{k}(n)$ is generated (as a Galois module) by $x \otimes 1$ and $\zeta_{l^{k}} \otimes 1$. (This strongly uses the cyclicity of the class group as a Galois module!) Both of these elements map to zero in the lower right hand corner, so this shows that the image of $\alpha_{\mathbb{Q}}$ is contained in the kernel of $\partial$, which is $K_{2 n}^{e t}\left(\mathbb{Z}\left[\frac{1}{l}\right]\right)\left[l^{k}\right]$. Since the map 
$\alpha_{\mathbb{Q}}$ was surjective to begin with, the upper vertical map in (d1) is an equality, and $\operatorname{im} \alpha_{\mathbb{Q}}=K_{2 n}^{e t}\left(\mathbb{Z}\left[\frac{1}{l}\right]\right)\left[l^{k}\right]=K_{2 n}^{e t}\left(\mathbb{Z}\left[\frac{1}{l}\right]\right)$.

It remains to see that the second generator $\alpha_{\mathbb{Q}}\left(\zeta_{l^{k}} \otimes 1\right)$ is superfluous. In fact it is zero. For $n=1$ this is a well-known property of symbols: before applying transfer, $\zeta_{l^{k}} \otimes 1$ maps to the symbol $\left\{\zeta_{l^{k}}, \zeta_{l^{k}}\right\}$, which is trivial by a simple calculation, For general $n$ we again get the trivial element already before applying transfer, as one sees by writing $\zeta_{l^{k}} \otimes 1 \in \mathcal{O}_{E}^{\times} \otimes \mathbb{Z} / l^{k}(n)$ as the cup product of $\zeta_{l^{k}} \otimes 1 \in \mathcal{O}_{E}^{\times} \otimes \mathbb{Z} / l^{k}$ with $1 \in \mathbb{Z} / l^{k}(n-1)$.

\section{The general construction}

We now remove the cyclicity assumption on our class groups. Moreover we will proceed in a more general way, descending only to $F^{+}$instead of all the way to $\mathbb{Q}$. (This could easily have been done in the previous part already, using character decomposition for the group $\Delta=\operatorname{Gal}\left(\mathbb{Q}\left(\zeta_{l}\right) / \mathbb{Q}\right)$.) Let us begin with an elementary algebraic lemma.

Lemma 3.1. Consider a presentation

$$
\left(\mathbb{Z} / l^{k}\right)^{s} \stackrel{\cdot A}{\longrightarrow}\left(\mathbb{Z} / l^{k}\right)^{s} \longrightarrow C \longrightarrow 0,
$$

where the matrix $A \in M_{s, s}\left(\mathbb{Z} / l^{k}\right)$ can be lifted to a matrix $\tilde{A} \in M_{s, s}\left(\mathbb{Z}_{l}\right)$ such that $d:=\operatorname{det} \tilde{A}$ divides $l^{k}$ in $\mathbb{Z}_{l}$. Then the kernel of the multiplication $\cdot A$ is the image of the multiplication by $B:=d^{-1} l^{k} a d(A):\left(\mathbb{Z} / l^{k}\right)^{s} \longrightarrow\left(\mathbb{Z} / l^{k}\right)^{s}$, where ad $(A)$ denotes the adjoint matrix of $A$.

Proof: Since $A \cdot a d(A)$ is $d$ times the identity matrix, we see that $A B$ is zero. (The quantity $d^{-1} l^{k}$ has to be read in $\mathbb{Z}_{l}$ of course.) Suppose conversely that $v \in \mathbb{Z}_{l}^{s}$ is a row vector, $\bar{v} \in\left(\mathbb{Z} / l^{k}\right)^{s}$ its image, and assume that $\bar{v}$ is in the kernel of $\cdot A$. Then $v \tilde{A}=l^{k} w$ with $w \in \mathbb{Z}_{l}^{s}$ and $v \tilde{A}=w l^{k} d^{-1} a d(\tilde{A}) \cdot \tilde{A}$, and $w^{\prime}=w l^{k} d^{-1}$ is in $\mathbb{Z}_{l}^{s}$. We may cancel $\tilde{A}$ and get $\bar{v}=w^{\prime} B$, so $\bar{v}$ is in the image of multiplication by $B$.

We now prepare the stage for an application of this lemma. Let $k$ be such that $l^{k}=\left|K_{2 n}^{e t}\left(\mathcal{O}_{F^{+}}\left[\frac{1}{l}\right]\right)\right|$, and recall that $E=\mathbb{Q}\left(\zeta_{l^{k}}\right)$. We denote by $X$ the minus part of the $l$-Sylow subgroup of the class group $C l\left(\mathcal{O}_{E}\right)$. Let $R$ denote the ring $\mathbb{Z}_{l}[\tilde{G}] /(1+j)$. Since $X$ is of projective dimension 1 (or 0 ) over $R$ (see [Sch]; the argument given there for prime cyclotomic fields works just as well in our case), and its Fitting ideal is the principal ideal generated by $\Theta_{*}$, we have a presentation of $X$ of the form

$$
R^{s} \stackrel{\cdot M}{\longrightarrow} R^{s} \longrightarrow X \longrightarrow 0,
$$

where $M \in M_{s, s}(R)$ and $\operatorname{det}(M)=\left(\Theta_{*}\right)$. Let $R_{0}=\mathbb{Z}_{l}[\Delta]^{+}$be the ring $\mathbb{Z}_{l}[\Delta] /(1-j)$, and let $\varepsilon$ be the natural projection from $\Lambda$ to $R_{0}$. Then $K_{2 n}^{e t}\left(\mathcal{O}_{F^{+}}\left[\frac{1}{l}\right]\right)$ has the same order as $\left[R_{0}: \varepsilon\left(\Theta_{*}^{(n)}\right)\right]$, by the class number formula already mentioned. Thus, if $A$ is $\varepsilon\left(M^{(n)}\right)$ taken modulo $l^{k}$, then $A$ is liftable to a matrix over $R_{0}$ whose determinant $d$ is $\varepsilon\left(\Theta_{*}^{(n)}\right)$; and since the index $\left[R_{0}: \varepsilon\left(\Theta_{*}^{(n)}\right)\right]$ has the exact value $l^{k}$, we obtain that $d$ divides $l^{k}$ in $R_{0}$.

Now let $\mathfrak{q}_{1}, \ldots, \mathfrak{q}_{s}$ be prime ideals of absolute degree 1 representing the generators of $X$ that correspond to the presentation (d2) of $X$. Let $S$ consist of these primes 
and the prime above $l$ in $E$. We consider the diagram:

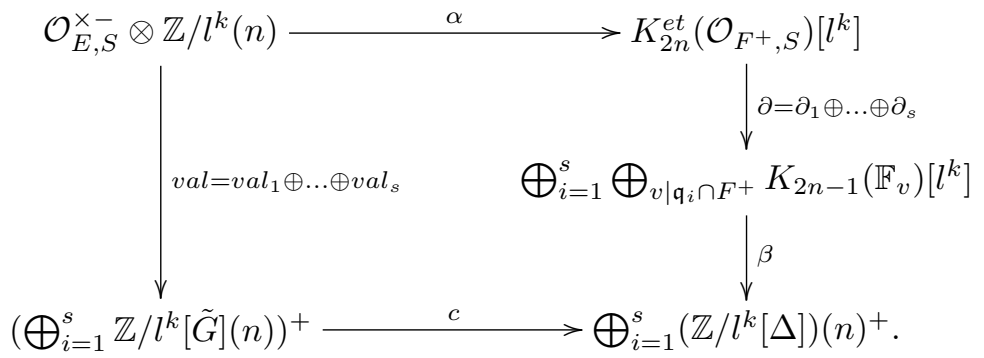

A few explanations: $v a l_{i}$ corresponds to $\mathfrak{q}_{i}$ exactly as $v a l$ corresponded to $\mathfrak{q}$ in the previous section. The map $\partial$ is the localisation map for the set of $F^{+}$-places below $S$ with the prime above $l$ removed; its $i$-th component goes to the direct sum of all $K_{2 n-1}\left(\mathbb{F}_{v}\right)$ with $v$ being conjugate to $\mathfrak{q}_{i} \cap F^{+}$. The map $\beta$ is similar as in the previous section; note that every $K_{2 n-1}\left(\mathbb{F}_{v}\right)\left[l^{k}\right]$ is again generated by $\zeta^{\otimes n}$, with $\zeta$ the image of $\zeta_{l k}$. More precisely, $\beta$ maps the tuple having $\zeta^{\otimes n}$ at position $\mathfrak{q}_{i} \cap F^{+}$and 1 everywhere else to the tuple having -1 at position $i$ and zero everywhere else. The map $c$ this time is canonically induced by taking $G$-coinvariants. Up to obvious technical changes (the main one being that transfer is not taken all the way down to $\mathbb{Q}$ but only to $F^{+}$) the argument of Lemma 2.2 proves that diagram (d3) is again commutative.

Now $\mathcal{O}_{E, S}^{\times-}$is generated up to roots of unity (which we may neglect later on, exactly as previously) by elements $x_{1}, \ldots, x_{s}$ satisfying

$$
\left(x_{i}\right)=\mathfrak{q}_{1}^{m_{i 1}} \ldots \mathfrak{q}_{s}^{m_{i s}}
$$

where the $m_{i j}$ are the coefficients of the matrix $M$ in the presentation (d2). We extend the diagram (d3) somewhat; $\iota$ maps the $i$-th standard basis vector to $x_{i} \otimes 1$.

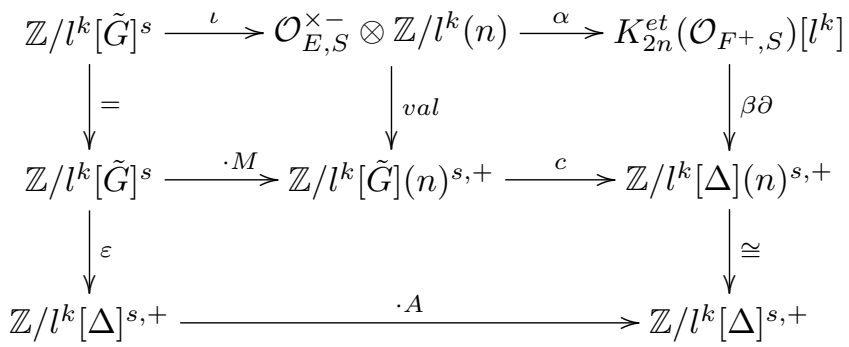

Then $\operatorname{val}\left(x_{i}\right)$ is simply the $i$-th row of $M$ read in $\mathbb{Z}_{l}[\tilde{G}]$ and modulo $l^{k}$. Therefore the upper left square commutes. Since $c$ is essentially taking coinvariants of the $n$-th twist, we get that $c\left(\operatorname{val}\left(x_{i}\right) \otimes 1\right)$ is the $i$ th row of the matrix $A$, and so the lower rectangle commutes as well.

We now obtain generators of $K:=K_{2 n}^{e t}\left(\mathcal{O}_{F^{+}}\left[\frac{1}{l}\right]\right)$ as follows:

$$
\begin{aligned}
K & =\alpha(\operatorname{ker}(c \circ v a l)) \\
& =\alpha \iota(\operatorname{ker}(c \circ v a l \circ \iota)) \\
& =\alpha \iota(\operatorname{ker}(c \circ(\cdot M))) .
\end{aligned}
$$

Now the map $\alpha \iota$ factors through the map $\varepsilon$ (which takes $G$-coinvariants), to give a map $\beta$. Thus $K=\beta(\operatorname{ker}(\cdot A))$. By Lemma 3.1, the kernel of multiplication of $A$ (the bottom horizontal map of the diagram (d4)) agrees with the image of multiplication by $l^{k} d^{-1} a d(A)$. Lifting this back through $\varepsilon$, we obtain the main result: 
Theorem 3.2. Let the matrix $M \in M_{s, s}(R)$ arise from a presentation of the l-part of the minus class group of $E$ as explained, and let $d \in \mathbb{Z}_{l}[\Delta]$ be the image of $\operatorname{det}(M)$ under $\varepsilon$ (twist $n$ times and send $G$ to 1$)$. We recall that $k$ is chosen such that the group $K_{2 n}^{\text {et }}\left(\mathcal{O}_{F^{+}}\left[\frac{1}{l}\right]\right)$ has order $l^{k}$. Let $B=\left(b_{i j}\right)$ be the adjoint matrix of $M$ multiplied by $l^{k} / d \in R_{0}$. Then the group $K_{2 n}^{e t}\left(\mathcal{O}_{F^{+}}\left[\frac{1}{l}\right]\right)$ is generated by the set of elements

$$
y_{i}:=\alpha\left(x_{1}^{b_{i 1}} \cdots x_{s}^{b_{i s}} \otimes 1\right), \quad i=1, \ldots, s .
$$

Remark 1: By very similar arguments we can prove that the relations between the $y_{i}$ 's are generated by the rows of $A$. Actually this is exactly what one expects, since the size of $K_{2 n}^{e t}\left(\mathcal{O}_{F^{+}}\left[\frac{1}{l}\right]\right)$ is predicted by the determinant of $A$. In fact one may show a priori using Iwasawa theory and codescent that the group $K_{2 n}^{e t}\left(\mathbb{Z}\left[\frac{1}{l}\right]\right)$ has a presentation of this type. What we have done is only to give an explicit description of the generators.

Remark 2: If Iwasawa's cyclicity conjecture holds (note that this conjecture is a consequence of Vandiver's conjecture), then we may take $s=1$, and everything becomes much simpler. This is the situation considered in the first part of this note.

\section{Acknowledgements}

The second author gratefully acknowledges the hospitality of AMU Poznań during two visits. The first author was partially supported by the Alexander von Humboldt Research Fellowship and an MNiSzW grant. The first author also thanks Centre de Recerca Matematica in Bellaterra for hospitality and support during a visit in summer 2008.

\section{References}

[BG1] G. Banaszak and W. Gajda, On the arithmetic of cyclotomic fields and the K-theory of $\mathbb{Q}$, Contemp. Math. 199 (1996), 7-18.

[BG2] _ Euler systems for higher K-theory of number fields, J. Number Th. 58 (1996), 213252.

[DF] W. Dwyer and E. Friedlander, Étale $K$-theory and arithmetic, Trans. Amer. Math. Soc. 292 (1985), 247-280.

[Ga] W. Gajda, On $K_{*}(\mathbb{Z})$ and classical conjectures in the arithmetic of cyclotomic fields, Contemp. Math. 394 (2004), 217-237.

$[\mathrm{Ku}]$ M. Kurihara, Some remarks on conjectures about cyclotomic fields and $K$-groups of $\mathbb{Z}$, Compositio Math. 81 (1992), 223-236.

[Sch] R. Schoof, Minus class groups of the fields of the l-th roots of unity, Math. Comp. 58 (1998), 1225- 1245 .

[Sou] C. Soulé, K-théorie des anneaux d'entiers de corps de nombres et cohomologie étale, Invent. math. 55 (1979), 251-295.

[Ta] J. Tate, Relations between $K_{2}$ and Galois cohomology, Invent. math. 36 (1976), 257-274.

[We] C. Weibel, Algebraic $K$-theory of integers in local and global fields, in: Handbook of $K$-theory vol. I, Springer 2005, 139-190

Department of Mathematics, Adam Mickiewicz University, 61614 Poznań, Poland

E-mail address: gajda@amu.edu.pl

FAKultät Informatik, Universität der Bundeswehr München, 85577 Neubiberg, Germany

E-mail address: cornelius.greither@unibw.de 